\title{
NOSOCOMIAL URINARY TRACT INFECTION DUE TO TRICHOSPORON ASAHII: A RARE CASE REPORT
}

Kumudini Panigrahi, Pragnya Paramita Panda, Basanti Pathi, Dipti Pattnaik, Sashi Shankar Behera

1. Assistant Professor, Department of Microbiology, Kalinga Institute of Medical Science, Bhubaneswar.

2. Assistant Professor, Department of Microbiology, Kalinga Institute of Medical Science, Bhubaneswar.

3. Assistant Professor, Department of Microbiology, Kalinga Institute of Medical Science, Bhubaneswar.

4. Professor, Department of Microbiology, Kalinga Institute of Medical Science, Bhubaneswar.

5. Associate Professor, Department of Obstetrics \& Gynecology, Kalinga Institute of Medical Science, Bhubaneswar.

\section{CORRESPONDING AUTHOR}

Dr. Kumudini Panigrahi

Asst. Professor, Dept of Microbiology

Kalinga Institute of Medical Science,

Bhubaneswar - 751024, Odisha, India

E-mail: kumudini_disha@yahoo.co.in.

Ph: 00919438116539.

ABSTRACT: Trichosporon asahii is a basidiomycetous yeast which causes white piedra and onychomycosis in immunocompetent hosts. In immunocompromised hosts this species may cause a number of localized and disseminated infections. Urinary tract infection by Trichosporon asahii is a rare occurrence .Few cases have been reported from India. We present a microbiologically confirmed urinary tract infection with T. asahii in a 52 year old diabetic, female patient who had undergone hysterectomy for dysfunctional uterine bleeding. Her urine sample was subjected to culture and sensitivity for postoperative rigor. Gram stain examination of the centrifuged urine revealed septate hyphae with arthroconidia and pus cells. Yeast like fungus was isolated in pure culture in three consecutive samples which was confirmed as Trichosporon asahii by standard tests. The response to antifungal therapy was dramatic. Trichosporon species though occasionally a part of normal flora, may be involved in causing nosocomial opportunistic infection more so in immunocompromised hosts. Very few cases of microbiologically confirmed urinary tract infection have been reported so far. Its diagnosis is likely to be missed or misinterpreted because of lack of awareness. Therefore T. asahii deserves recognition as a pathogen.

KEY-WORDS: Trichosporon asahii, Urinary tract infection

INTRODUCTION: Trichosporon is a genus of anamorphic yeasts with distinct morphological characters of budding cells and true mycelium that disarticulates to form arthroconidia. ${ }^{1}$ It is present in the external environment and is isolated mainly from the soil, water, air and organic materials. ${ }^{2}$ In the past $\mathrm{T}$. bigelli was known to be the sole pathogenic species under Trichosporon genus, responsible for superficial infection of skin such as white piedra and infection of nails. Following extensive taxonomic re-evaluation presently seven species are known to be pathogenic for human beings. They include Trichosporon asahii, Trichosporon asteroids, Trichosporon cutaneum, Trichosporon inkin, Trichosporon mucoides, Trichosporon ovoides, and Trichosporon louberieri of which Trichosporon asahii causes most of the disseminated infections. ${ }^{3}$

Disseminated infections are increasingly reported in immunocompromised hosts. ${ }^{3}$ 
Trichosporonosis manifests as fever, pulmonary infiltrates, azotemia, renal dysfunction and skin lesions. The yeast can be isolated from sputum, urine, skin and blood. Trichosporon species are important cause of sepsis in immature infants, and summer type hypersensitivity pneumonitis (SHP) in Japan. ${ }^{2}$

CASE PRESENTATION: A fifty two year old female patient was admitted to our Gynaecology ward in August, 2011 for dysfunctional uterine bleeding. As per her previous medical records, she was a known diabetic and hypertensive without any history of tuberculosis or HIV. On USG abdomen and pelvis, multiple myomas were found in the uterus, for which hysterectomy was done. Post operatively patient was with catheter and on intravenous Ceftriaxone and Amikacin She was responding well to the management until fifth day. On sixth day of surgery, she developed spiky fever associated with chill and rigor. Her blood parameters were as follows: heamoglobin: $9.2 \mathrm{gm} / \mathrm{dl}$, Total leucocyte count 14,200/ $\mu$ l, Fasting and postprandial blood sugars were $190 \mathrm{~g} / \mathrm{dl}$ and $230 \mathrm{gm} / \mathrm{dl}$ respectively. Her blood Urea and creatinine were $47 \mathrm{mg} / \mathrm{dl}$ and $2.2 \mathrm{mg} / \mathrm{dl}$ respectively.

The patient's urine sample was sent for routine microscopic examination, aerobic culture and sensitivity testing. Routine microscopic examination of urine showed numerous pus cells. The Gram stain examination of the patient's centrifuged urine sample showed plenty of pus cells and septate hyphae with arthroconidia. The urine sample was inoculated with a standard loop on CLED (Cystine Lactose Electrolyte deficient) agar and incubated overnight at $37{ }^{\circ} \mathrm{C}$. Tiny, creamy - white, dry and wrinkled colonies were seen on CLED agar (Figure 1). The Gram stain of the colony revealed septate hyaline hyphae with arthrospores and few budding yeast cells (figure 2). The colony was subcultured on a set of Sabouraud's Dextrose Agar (SDA) and incubated at $28{ }^{\circ} \mathrm{C}$.and $37{ }^{\circ} \mathrm{C}$. At both these temperatures, colonies of yeast like fungus (figure 3) were obtained in pure cultures with in $24 \mathrm{hrs}$. Routine examination of urine and culture on SDA of two more consecutive samples revealed the similar findings as that of the first. The yeast was identified to be $\mathrm{T}$. asahii with cornmeal agar morphology (Figure 4), hydrolysis of urea, carbohydrate fermentation -assimilation test and nitrate assimilation test.

Based on the culture report, antifungal therapy with Fluconazole was initiated and the general condition of the patient improved dramatically. Within four days of therapy, other complains were resolved. After 4 weeks of antifungal treatment, the urine sample was sent for repeat fungal culture and it was found to be negative for fungus.

DISCUSSION: Trichosporonosis is usually an insidious disease but it can present as an opportunistic infection in susceptible hosts. Trichosporon species are occasionally a part of normal flora of human skin. ${ }^{2}$ This yeast has been documented on intact perigenital skin in 12.4 $\%$ of population.$^{2}$ Factors that enhance the mucosal colonization and subsequent invasion of the fungus include broad spectrum antibiotic treatment and break in mucosal barrier. ${ }^{4}$ Candida species is well known to cause infection in diabetic patients. ${ }^{5}$ Trichosporon asahii causes white piedra, a superficial infection of hair shaft mainly restricted to tropical regions and onychomycosis in immunocompetent humans. ${ }^{6}$

In the past few decades, a worldwide increase in the incidence of opportunistic fungal infections has been observed. ${ }^{7,8}$ The increase in immunocompromised state has been accompanied by an increase not only in frequency of opportunistic fungal infections but also in the variety of species involved. ${ }^{5}$ Among them, deep seated trichosporonosis is a lethal opportunistic infection found in immunocompromised patients, particularly those who are 
neutropenic due to cytotoxic therapy for heamatological malignancies ${ }^{7,8} \mathrm{In}$ immunodeficient host it has been isolated from blood ,skin, urine and viscera causing various localized or disseminated deep infections. ${ }^{6}$

UTI due to Trichosporon asahii is a rare occurrence. Very few cases of microbiologically confirmed infections have been reported so far., ${ }^{3,5}$ Though reported rarely, Trichosporon asahii is a known pathogen to cause UTI. It has been reported to cause UTI in seven month old child following urethral valve surgery from India. ${ }^{5}$ Nosocomial infection due to trichosporon asahii has been reported from chile. ${ }^{9}$

We isolated T. asahii from the urine of a fifty two year old, catheterized, postoperative, diabetic lady who had undergone hysterectomy for dysfunctional uterine bleeding.

Isolation of the same yeast in three consecutive urine samples in significant counts and the fact that no bacteria was isolated, establishes Trichosporon asahii as an etiological agent of urinary tract infection. Besides this, the clearance of the organism from the urinary tract with recovery of the patient following antifungal treatment strongly associates the yeast as causative agent of UTI.

Our patient exhibited risk factors such as trauma during surgery, presence of indwelling catheter, use of broad spectrum antibiotics and more over she was a known diabetic. It is possible that the organism colonized the catheter from the human flora during catheterization and subsequently causing invasion and UTI.

CONCLUSION: Trichosporon asahii is a basidiomycetous yeast forming yeast like budding cells along with true mycelia and arthroconidia. It grows in SDA at $24^{\circ} \mathrm{C}$ and $37^{\circ} \mathrm{C}$ with in $24 \mathrm{hrs}$. It produces arthrospores in corn meal agar. T. asahii is responsible for causing white piedra and onychomycosis in immunocompetent hosts. It has been associated with causation of various localized and disseminated invasive infections in immunocompromised hosts. Cases of T. asahii infections are on the rise in immunocompromised and hospitalised patients. UTI by T. asahii has rarely been reported. Its diagnosis is likely to be missed or misinterpreted because of unawareness. Therefore T. asahii deserves recognition as a pathogen.

\section{REFERENCES:}

1. Middelhoven WJ:Identificetion of clinically relevent Trichosporon species. Mycoses 2003; 46:45-9.

2. Pini G, Faggi E, Donato R, Fanci R: Isolation of Trichosporon in a hematology word. Mycoses 2005; 48: 45-9.

3. Chowdhary Ahmad S, Khan ZU, Duval DC, Randhawa HS: Trichosporon asahii is an emerging etiological agent of disseminated trichosporonosis: A case report and update. Ind J Med Microbiol 2004; 22: 16-22.

4. Wolf DG, Falk R, Hacham M, Theelen B, Boekhout T, Scorzetti G, et al.: MultidrugResistant Trichosporon asahii infection of Nongranulocytic patients in Three Intensive Care Units. J Clin Microbiol 2001; 39: 4420-5.

5. Sood, Pathak D,Sharma R, Rishi S : Urinary tract infection by Trichosporon asahii. Ind J Med Microbiol 2006; 24(4) : 294-6.

6. Panagopoulou P, Evdoridou J, Bibashi E, Filioti J, Sofianou D, Kremenopoulos G, et al: Trichosporon asahii an unusual cause of invasive infection in neonates. Pediatr Infect Dis Jr 2002; 21:169-70. 
7. Anaissie EJ: Opportunistic mycoses in the immunocompromised host: experience at a cancer center and review. Clin Infect Dis 1992;14:43-53.

8. Girmenia C, Pagano L, Martino B, D'Antonio D,Fancir Specchia G, Melillo L, et al : Gimema infection programm. Invasive infections caused by Trichosporon species and Geotrichum capitatum in patients with hematological malignancies: A retrospective multicentric study from Itally and review of the literature. J Clin Micrbiol 2005; 43:1818-28.

9. Silva V, Zepeda G, Alvareda D: Nosocomial urinary tract infection due toTrichosporon asahii. First two cases in Chile.Rev Iberoam micol 2003; 20: 21-3.

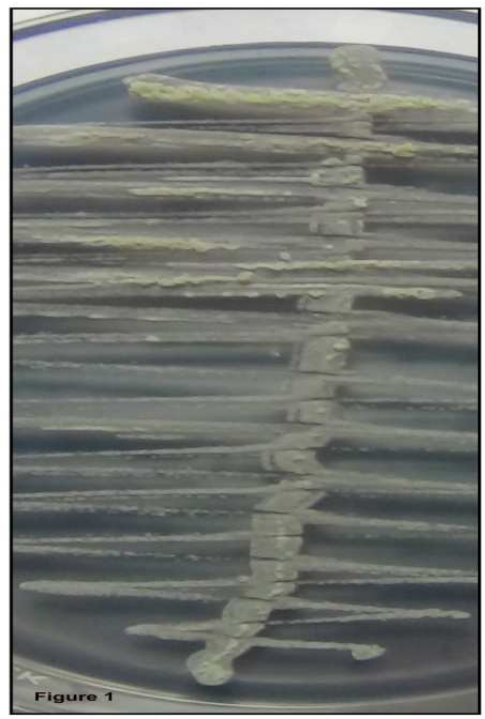

FIGURE 1: Colony morphology of the isolate on CLED agar, after $24 \mathrm{hrs}$ of aerobic incubation at $37 \mathrm{deg} \mathrm{C}$.

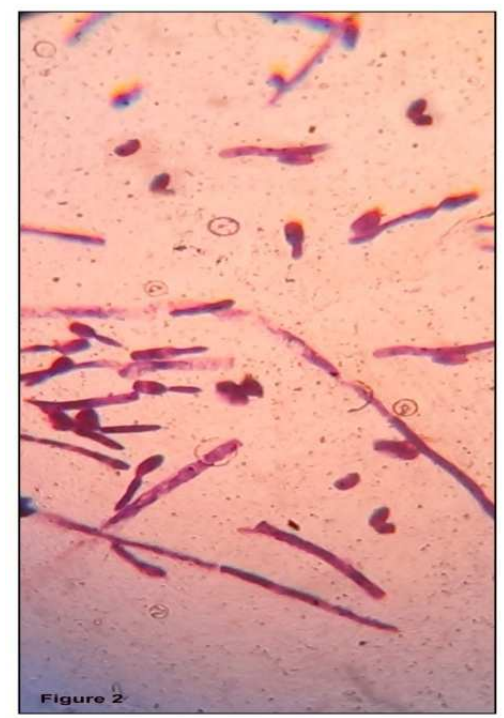

FIGURE 2: Gram stain morphology of the isolate,

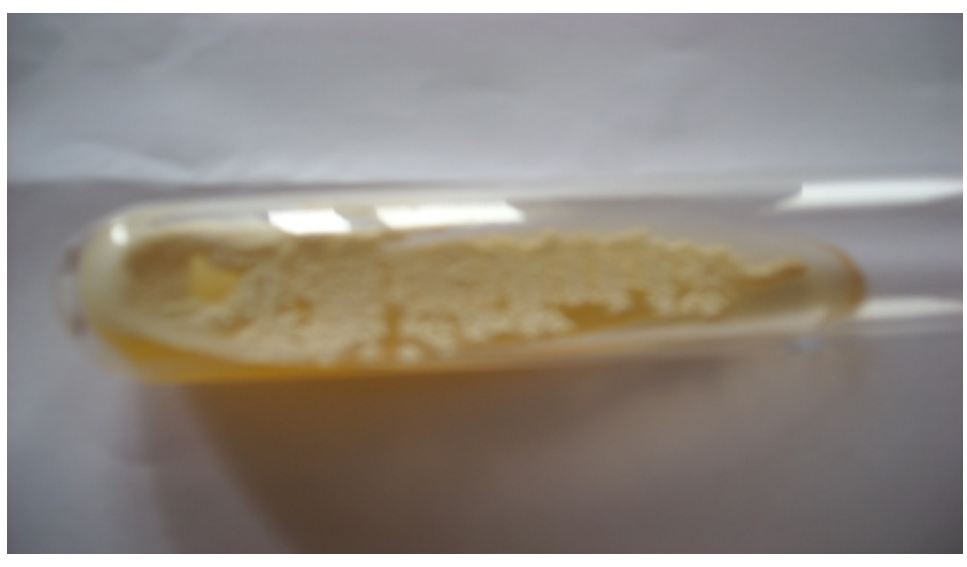

FIGURE 3: Colony morphology on SDA tube after $24 \mathrm{hrs}$ of aerobic incubation at $37 \mathrm{deg}$ C. 


\section{CASE REPORT}

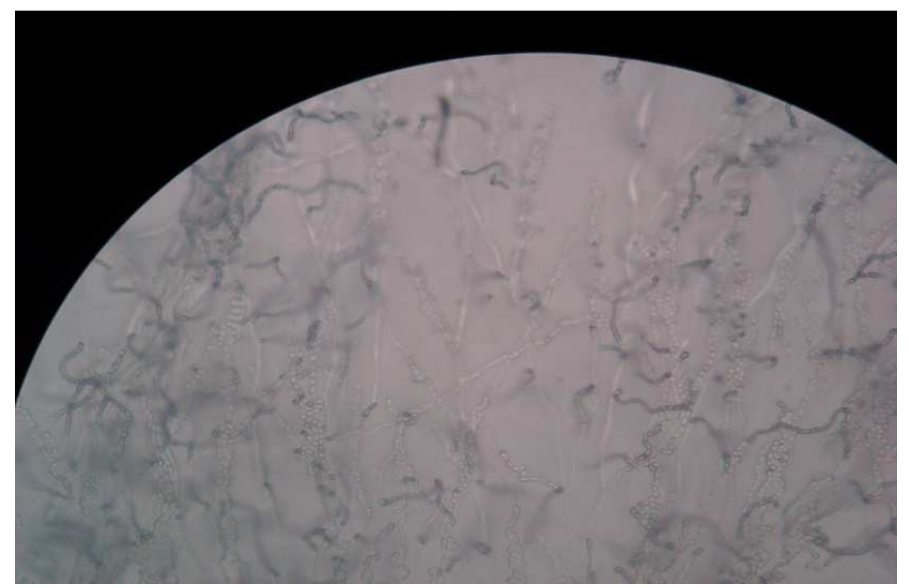

FIGURE 4: Corn meal agar morphology after $48 \mathrm{hrs}$ of incubation 